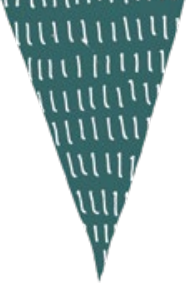

\title{
(HAPTER 5:
}

\section{HEAITH ECONOMIOS}

\section{Linda Davies and Gemma Shields}

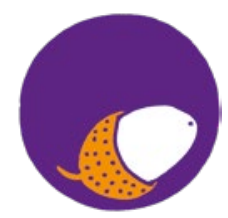

\section{(HAPTER OVERVIEW}

Evidence is needed to inform and guide the choices that healthcare organisations make in relation to how budgets are spent. The associated costs and benefits of health treatments are key components of such decisions. An economic evaluation is a way of systematically identifying the costs and benefits of different health activities and comparing these to make an informed decision about the best course of action based on the evidence available. Economic evaluations can also be used to identify uncertainty around the likely costs of a particular health activity and to compare this against a 'willingness to pay' threshold, in order to judge their value for money. Economic evaluations can be done as part of randomised controlled trials or can draw on evidence taken from other sources (e.g. surveys). Similar to clinical evidence, economic evidence needs to be updated and researched as new questions arise or more evidence becomes available.

\section{LEARNING OBJECTIVES}

By the end of this chapter you should be able to:

1. Understand why economic evaluations are needed

2. Understand the key parts of economic evaluations and the data that feed into them

3. Be able to begin to interpret and understand the results of economic evaluations 


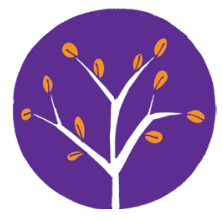

\section{INTRODUCTION}

The cost of providing healthcare is rising. As the population grows and becomes older, this increases the demand for healthcare interventions (e.g. drugs, therapies and services). Health services have limited budgets to meet these demands and need fair and objective ways of deciding which treatments, out of all those available, offer the best value for money. Economic evaluations compare the costs and benefits of a healthcare approach to assist decision makers in making these decisions.

The results of economic evaluations help decision makers make choices between the many healthcare interventions available which in turn helps to ensure that resources are used in the most efficient way to maximise the health of a particular patient group or population. Economic evaluations bring together data on healthcare benefits and costs, as well as identifying any uncertainty that might exist in the research data. In this chapter we will explore the role of economic evaluation in healthcare, the different types of evaluations that might be conducted and how economic data can be analysed.

\section{Making choices in healthcare}

First, let's consider how we make choices in our everyday lives - we consider a number of different things, including the likely benefits and drawbacks of our choice, its associated costs and risks, and who it might involve. We also consider what we do not know but might need to know, and, if necessary, seek further information.

For example, consider buying a car. If you have more than one option, you might compare cars in terms of their costs (and whether or not these are affordable), their mileage, makes, models and from whom or where you are buying them. You will consider your options, weigh up these different pieces of information and decide which one, if any, is best for you overall. We make many decisions every day in the same way (e.g. which brand should I buy in the supermarket? How should I travel to work?).

Choices about healthcare differ from these more 'everyday' choices in a number of ways:

1. The individual receiving the care doesn't always pay (In the UK for example, the individual receiving the care doesn't always pay in full). 


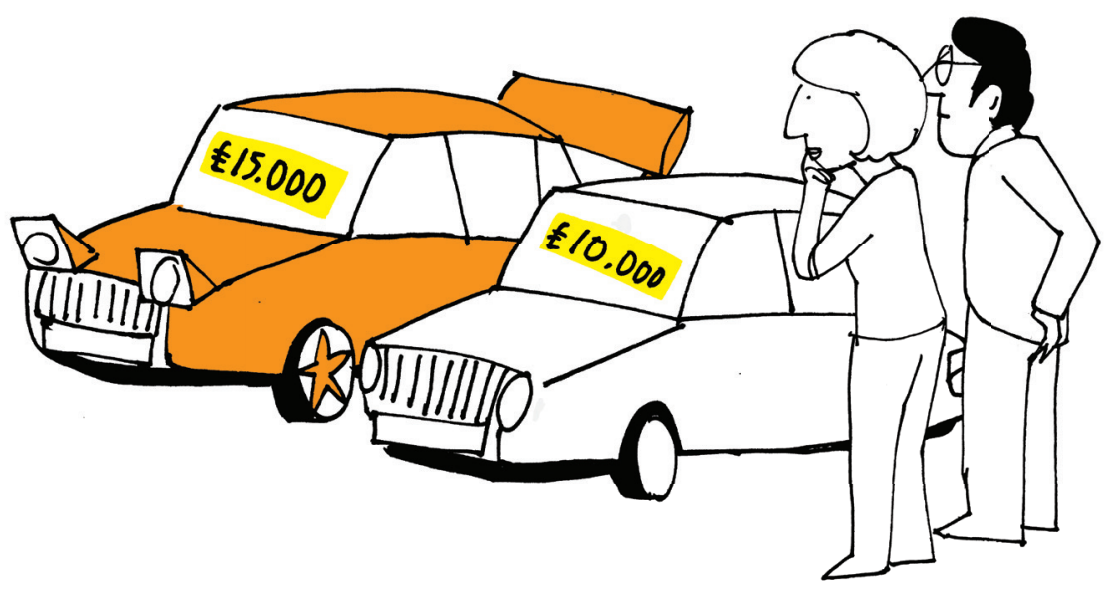

2. Choices about treatment availability are often made before a treatment is needed. National organisations will look at a range of evidence (e.g. evidence of treatment effectiveness, side effects and costs), to judge which treatments should be made available and recommended for use.

3. Treatment choice is influenced not only by the person who will receive the treatment, but also by their healthcare providers (e.g. your doctor will likely prescribe a treatment for you, based on the evidence and their knowledge).

\section{The role of an economic evaluation}

Generally speaking a treatment that is shown to be beneficial to service users (e.g. reducing symptoms or improving quality of life) whilst at the same time having low costs for the organisation (e.g. financial and time) would be considered the best approach.

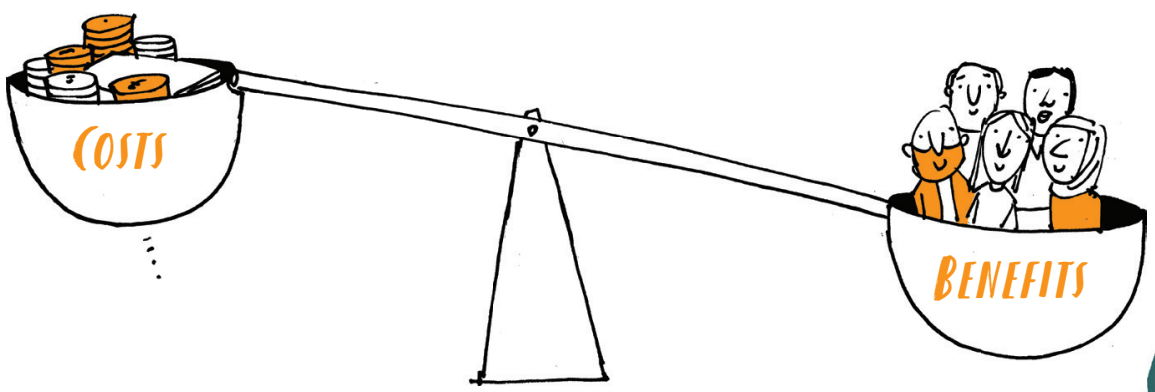


In this chapter, we will focus on cost-effectiveness and cost-utility evaluations, as these are the most recommended methods for economic evaluations in England (NICE method guide, 2013).

Cost-effectiveness evaluation: This type of analysis looks at differences in the costs and health benefits of two or more interventions. It summarises this difference by producing something called 'a cost per health benefit'. Health benefits are usually measured in natural units that are relevant to an individual, e.g. life -years or the number of symptom free days obtained with treatment. A cost-effectiveness evaluation usually compares one (newer) treatment with another (older or more routine treatment), to see how much cost would need to be spent by the health service to extend life by one -year. A more cost-effective treatment would allow patients to gain the same health benefit at less cost. Cost-effectiveness analysis provide very useful information but can only explore the cost of achieving one type of health benefit at a time.

This means that any other benefits obtained from the treatment, or the value placed on that benefit by an individual, may not be taken into account.

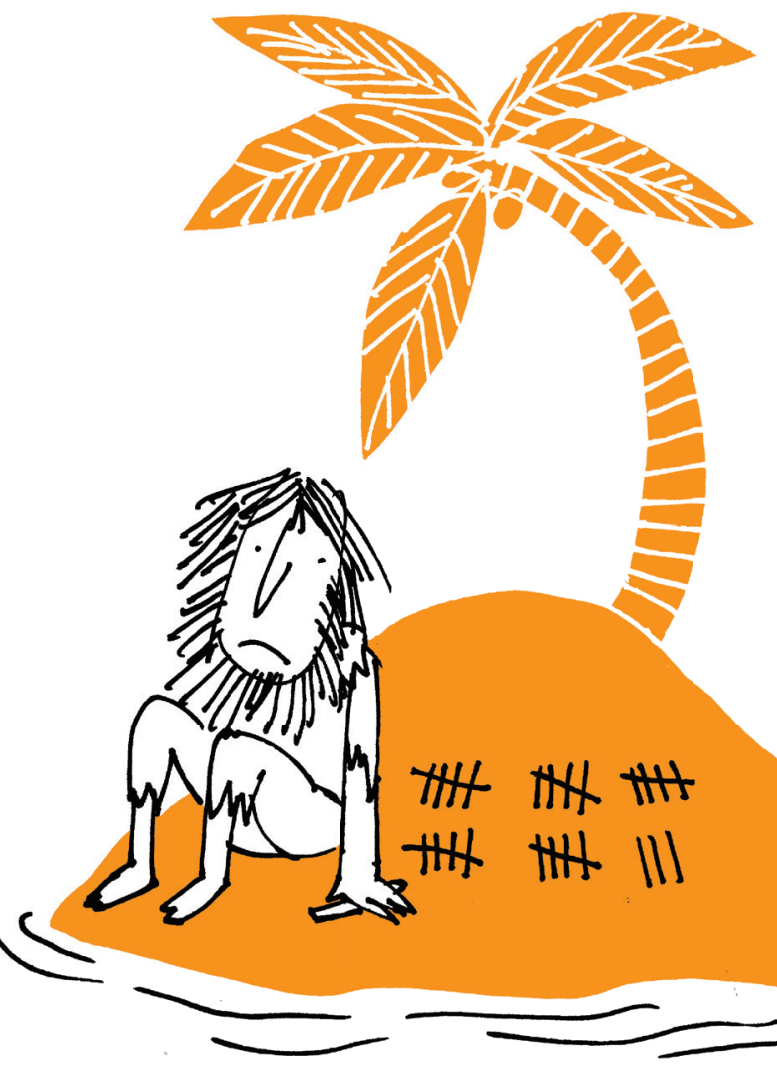

Cost-utility evaluation: $A$ costutility evaluation is a type of costeffectiveness analysis but is different because it is able to take into account more than one health benefit (e.g. symptom free days). Instead, the health benefit measure used, referred to as 'utility', takes a wider approach, often also incorporating quality of life. This is important because although a costeffectiveness study can tell you that a treatment may prolong a person's life by one-year, it does not allow you to consider the quality of that life year for the individual. Cost -utility analyses allow you to look at both the quantity and quality of any health benefits. 
In the UK, the National Institute for Health and Care Excellence (NICE) evaluates new health interventions. NICE states that the health effects of these new treatments should always be expressed as quality-adjusted lifeyears (QALYS). A QALY is a measure of both quantity of life gained, and the quality of the health that is achieved. One QALY is equal to one year of life in perfect health. QALYs are calculated by estimating the years of life available to a patient following a particular treatment, and adjusting that to take into account the quality of those years of life for an individual patient. Researchers do this by asking people about how well they undertake daily activities such as washing and dressing, how well they can get around and the pain they experience from mental or physical health symptoms.

\section{Defining healthcare costs}

Economic evaluations bring together data on healthcare benefits and costs. The type of costs required to undertake an economic evaluation in healthcare include:

- Direct costs - the resources needed to treat an episode of illness, to monitor changes in health or to prevent health problems for example time and salary cost of a community mental health nurse.

- Indirect costs - the wide range of other costs that may be affected by a person's health, for example the costs that result from not being able to work while unwell (e.g. lost wages).

\section{Example}

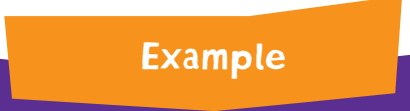

There are two groups of antipsychotics that are used to treat schizophrenia; First-Generation Antipsychotics (FGA) and Second-Generation Antipsychotics (SGA). First Generation Antipsychotics are an older type of antipsychotic medication than Second Generation Antipsychotics. In 1999, a trial began to compare the use of FGA versus SGA. This trial was called the CUTLASS trial.

At the time the CUTLASS trial began, doctors could only prescribe first generation drugs. However local psychiatrists and service users wanted the newer second generation drugs to be made available, as there was some evidence that they were safe to use and were effective in reducing the symptoms of schizophrenia, with less troublesome side-effects. 
However there was a big cost difference; the newer SGA drugs cost the NHS around $€ 1500-€ 2000$ per person per year of treatment, compared to around $€ 100$ a year for the FGA.

The research trial aimed to answer the following questions:

1. Should health services have the option to provide SGA?

2. Should SGA replace any or all of the FGA?

To do this, the trial looked for any differences between FGA and SGA in relation to:

their impact on people's health (e.g. physical and mental health symptoms)

their side effects

the costs of using the medication

their cost-effectiveness

the certainty that the results were correct.

Only direct costs were included. This covered the costs of providing the two types of antipsychotic medication and the costs of any other health services that were received (e.g. inpatient, outpatient and community care). The benefits of the FGA and SGA were summarised as QALYS, calculated by looking at differences in survival and quality of life.

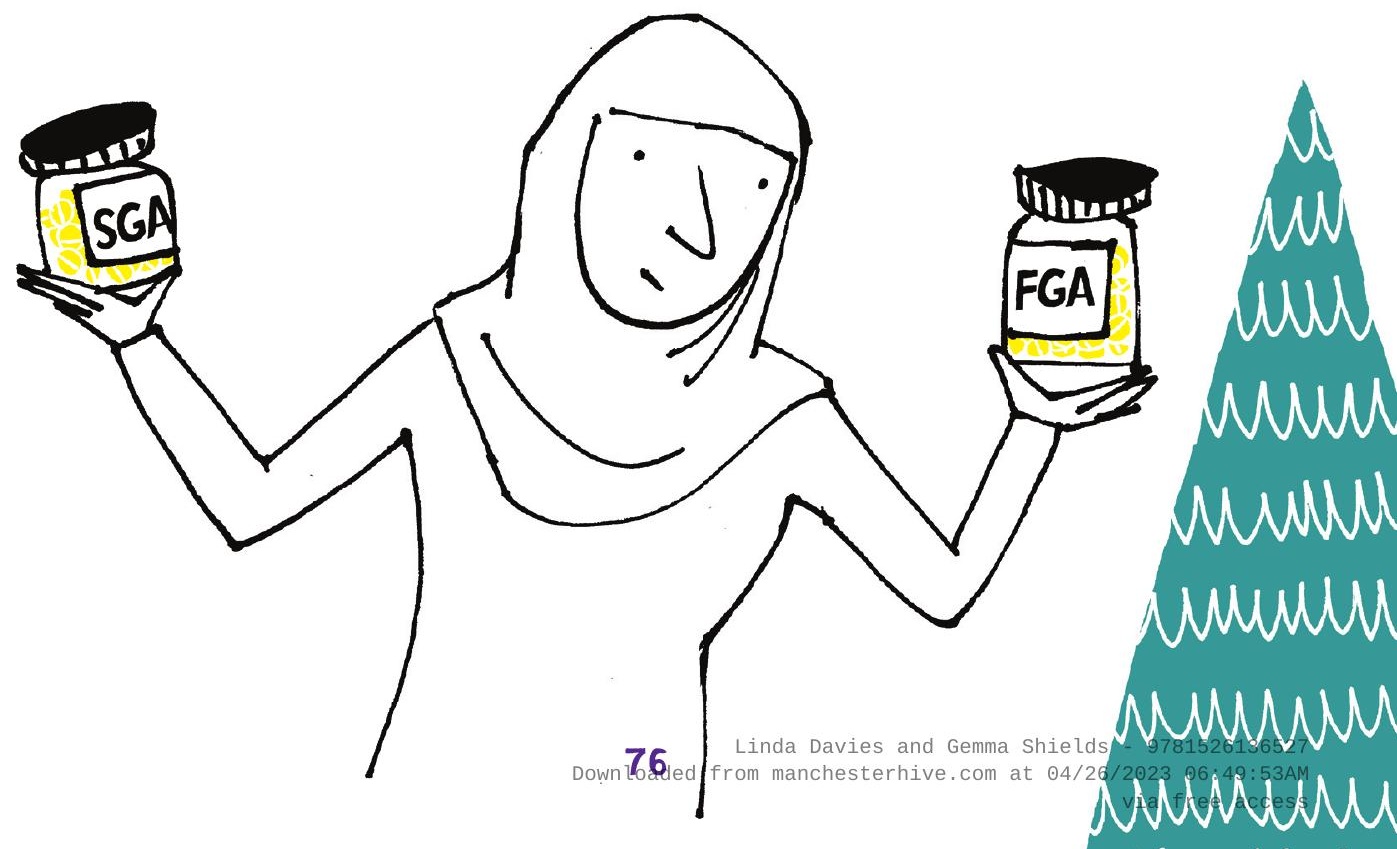




\section{Conducting an economic evaluation}

Figure 14 provides a visual summary of a cost-effectiveness or cost-utility economic evaluation. The first box shows that decision makers are often faced with a choice. In our example, the choice was between two groups of drugs for schizophrenia (FGA vs SGA).

By comparing the costs of both options and their health benefits, the economic evaluation produces an important result. This result is called an Incremental Cost-Effectiveness Ratio (ICER). Although this sounds very technical, it is simply the difference in the costs of the two treatments divided by the difference in benefit. The ICER gives you the cost per health benefit. What the health benefit is depends very much on whether you are doing a cost-effectiveness analysis or, e.g. you could have calculated the cost of each symptom free day gained or a cost-utility nalysis e.g. the cost of a quality adjusted life year (QALY).

\section{Figure 14 Diagram of cost-effectiveness}

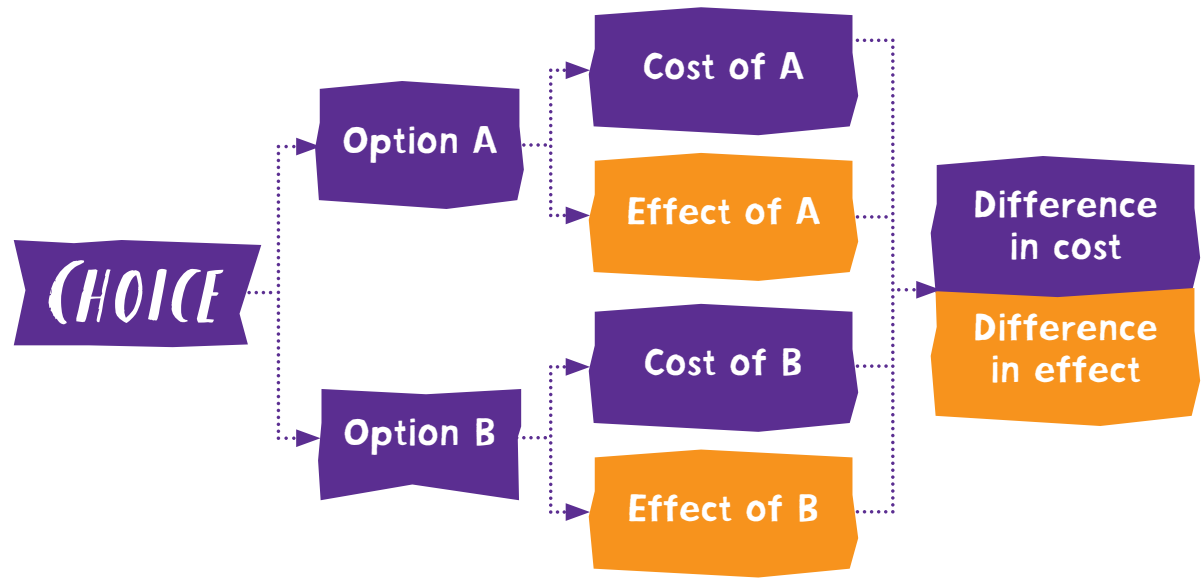




\section{Willingness to pay}

Often the cost of the health benefits identified through an economic analysis will be compared with the maximum amount that a healthcare organisation is prepared to pay to improve the health of its community (known as the willingness to pay threshold - WTPT). This helps to determine if a new intervention offers any demonstrable value over an old one, and whether it should be used. Willingness to pay thresholds are often decided in advance by organisations and reviewed annually. For example, NICE guidelines refer to a threshold of $€ 20,000-€ 30,000$ per QALY. Therefore, hypothetically speaking, if a new drug treatment was found to cost the NHS £11,000 a year in order for a service user to gain one additional QALY then it would be considered cost effective in line with NICE guidelines. It could then be considered a viable option for policy makers and service providers.

An example of this type of analysis is shown in Figure 15. If a new intervention is found to be both cheaper and more effective, it is said to 'dominate' the comparator (usual care) and lies in the bottom right-hand quarter. If it is more expensive and less effective it will instead be' dominated by' the comparator and fall in the top left quadrant. ICERs in the remaining quadrants need to be judged against the pre-determined willingness to pay threshold. ICERs falling under the line will be judged to be cost effective, whereas ICERs above the line will not.

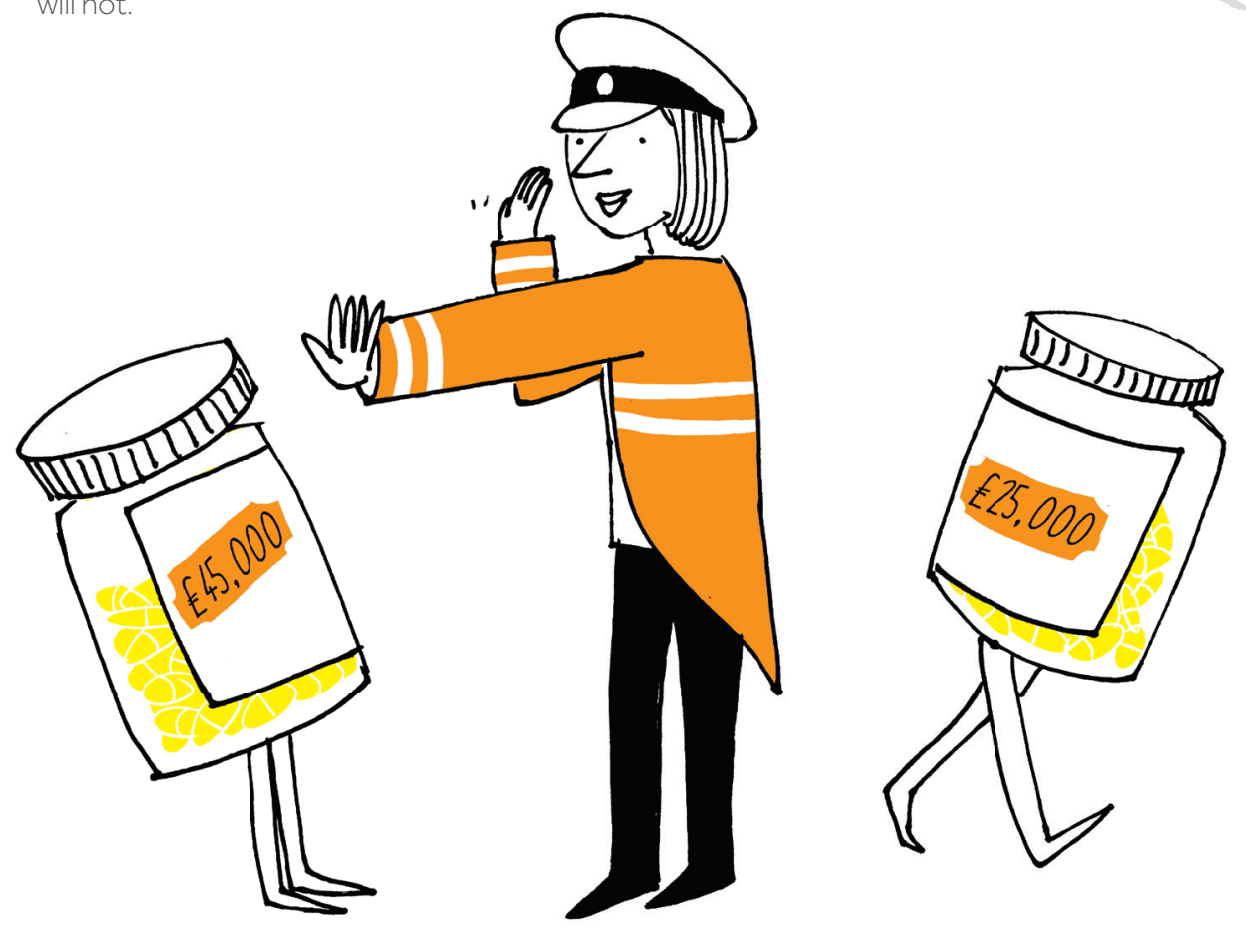


Key: WTPT, willingness to pay threshold.

In the CUTLASS trial, FGAs were found to cost slightly less than SGA ( $€ 18,858$ vs. $€ 20,118$ ). They were also found to marginally increase QALYs (0.74 vs. 0.67). This means that, on paper at least, the first generation drugs look like the best choice for health services to provide. However, because the differences in costs and benefits were relatively small, another possible option might be to offer both types of drug and let service users decide for themselves which medication they prefer. It is important to remember the ICER result is at best an estimate, and a level of uncertainty will always be present in the data. For this reason the ICER is an important, but not the only, consideration in commissioning health services and many other factors, such as patient need and experience can influence decision making.
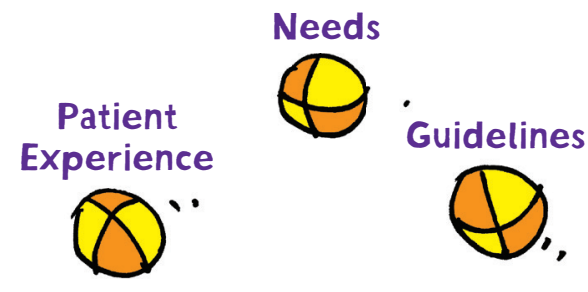

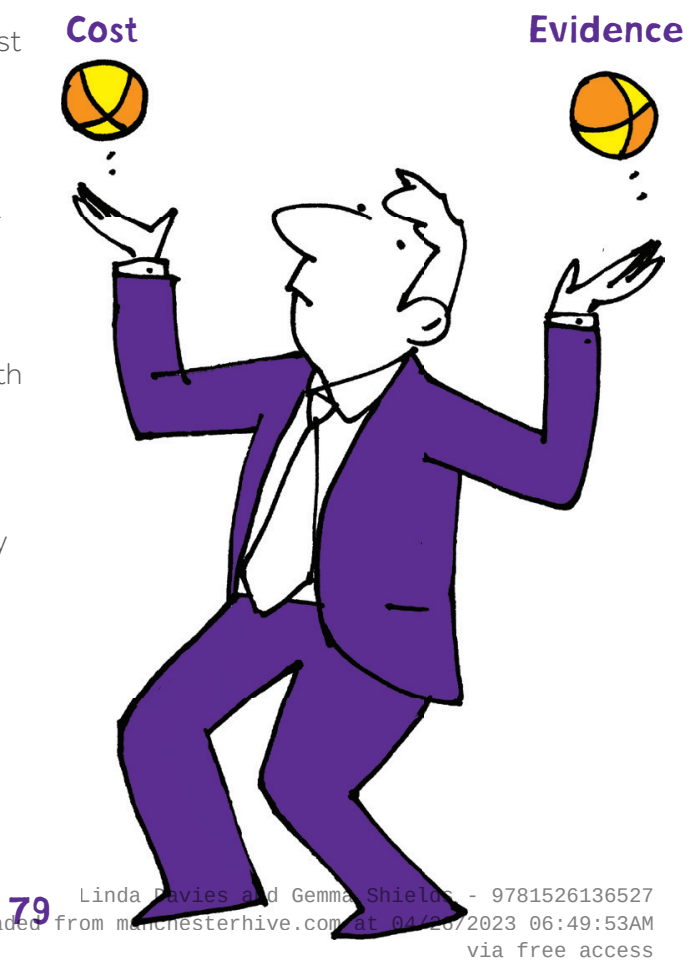




\section{EXAMPLE}

\section{TABLE 3 CUTLASS TRIAL ECONOMIC EVALUATION RESULTS}

\begin{tabular}{|c|c|c|c|}
\hline $\begin{array}{l}\text { One year costs } \\
\text { and QALYs }\end{array}$ & FGA & SGA & $\begin{array}{l}\text { Net value } \\
\text { (FGA minus } \\
\text { SGA) }\end{array}$ \\
\hline Cost of all services & $€ 18,858$ & $€ 20,118$ & $-€ 116$ \\
\hline QALYs & 0.74 & 0.67 & 0.04 \\
\hline
\end{tabular}

\section{Acknowledging uncertainty in economic evaluations}

Estimating costs and health benefits is not an exact science and therefore there is always some uncertainty around the data informing an economic evaluation. Estimates of costs and benefits may differ across populations and healthcare providers. Plotting the cost-utility results produced with different estimates of costs and effects can help to judge whether or not an intervention is, on balance, likely to be cost-effective. In a similar vein, it is important that economic evaluations are critically evaluated to ensure that they are robust and relevant to decision makers. Like trials, economic evaluations can be prone to bias and this can raise the level of uncertainty associated with their findings.

It is very important that economic evidence, like any other research evidence, is updated regularly as and when decision and policy makers have new questions, and as healthcare systems, population needs and treatment options evolve. The research cycle (shown in Figure 16) describes how economic data, like other forms of data can contribute to the flow of knowledge and new research aims.

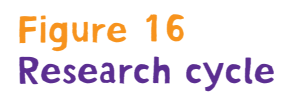

\section{Identify policy}

or practice issue

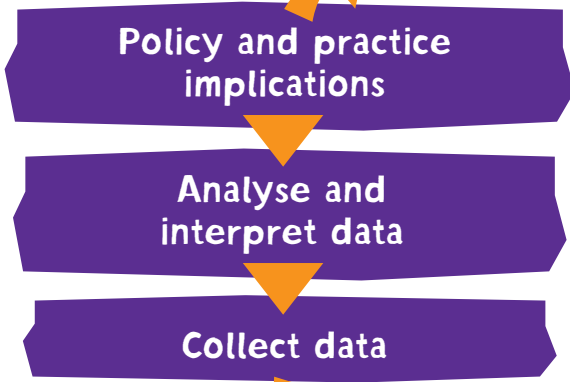

Specify the research question

Assess economic evidence

Identify need for further evaluation 


\section{PPI stories from EQUIP}

Next, Andrew describes his experiences on being involved in the health economic elements of the EQUIP project.

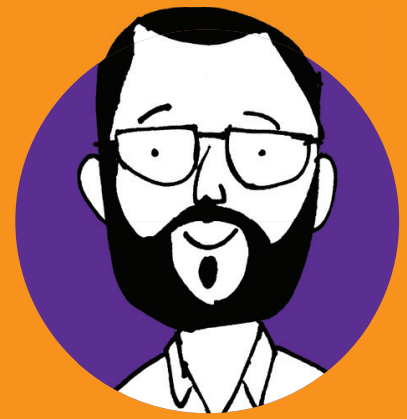

\section{ANDREN'S STORY}

The EQUIP health economists devised a survey to try to capture service user's and carer's preferences for involvement in care planning. The economists needed to collect a lot of detailed information to do this, which meant the survey was not always straightforward to understand or complete.

It was incredibly important that I and my colleagues were involved in this aspect of the EQUIP study as we were able to contribute a lot.

We produced a lay-summary of the study's aims, and we piloted the survey. which gave us a good idea of how long it would take to complete, and what issues might be encountered in its completion. We revised the survey where we could to make it as user-friendly as possible. We attended outpatient clinics to give out the study packs and assist people in completing the survey where needed. We also utilised our existing networks, including social media and our contacts with local service user and carer groups, to publicise the study. Together, these activities help the study team to collect all the data that they needed. 


\section{REFLETTIVE EXERCISE}

Think of a healthcare technology and imagine that you are designing an economic evaluation for that technology.

What would it be compared to?

- What costs would you include in the economic evaluation?

- What health benefits would you include in the economic evaluation?

\section{REFTRENCIS AND FURIHER READING}

For interested readers who want to learn about economic evaluation in more detail, we recommend:

Drummond, Michael F., Sculpher, Mark J., Claxton, Karl, Stoddart, Greg L., and Torrance George W (2015). Methods for the economic evaluation of health care programmes. Oxford University Press. 